\title{
BISE REPORIS
}

https://doi.org/10.23873/2074-0506-2020-12-4-311-318

Использование стент-графта для зндоваскулярного лечения ложной аневризмы, возникшей после нефрэктомии трансплантата

\author{
C.А. Прозоров \\ ГБУЗ «НИИ скорой помощи им. Н.В. Склифосовского ДЗМ», \\ 129090, Россия, Москва, Большая Сухаревская пл., д. 3
}

Автор, ответственный за переписку: Сергей Анатольевич Прозоров, д-р мед. наук, ведущий научный сотрудник отделения лучевой диагностики НИИ скорой помощи им. Н.В. Склифосовского,

surgeonserge@mail.ru

\section{Аннотация}

Введение. Сосудистые осложнения после небрэктомии трансплантата бъвают редко. Цель сообщения анализ эфббективности имплантации стент-грабта при лечении ложной аневризмь, возникшей после нефьрэктолии трансплантата.

Клинический случай. Мужчине 50 лет за последние 23 года бъли выполненъ три трансплантации трупной почки и два удаления трансплантата. Через 12 лет после последней небрэктолии трансплантата он поступил с жалобами на боли в животе и наличием пульсирующего образования в левой подвзооной области. Проведеннъе компьютерная томографическая ангиограбия, ультразвуковое исследование и ангиограбия показали наличие ложной аневризмы левой наружной подвздошной артерии. Илплантаиия стент-графбта позволила решить возникшую проблему с хорошими клиническим и ангиограбическим результатами.

Заключение. Лечение в таких случаях возможно как при открытой операции, так и с использованием эндоваскулярных методов. Хирургический метод сопровождается высоким риском осложнений, тогда как әндоваскулярное лечение имеет преимущество минималъно инвазивного метода с низкой кровопотерей.

Ключевые слова: трансплантация почки, ложная аневризма, стент-графт

КонФликт интеРЕсов Автор заявляет об отсутствии конфликта интересов

Финансирование Иследевание проводилось без спонсорской поддержки

Для цитирования: Прозоров С.А. Использование стент-графта для эндоваскулярного лечения ложной аневризмы, возникшей после неррэктомии трансплантата. Трансплантология. 2020;12(4):311-318. https://doi.org/10.23873/2074-0506-2020-12-4-311318 


\title{
Endovascular treatment of the pseudoaneurysm using stent-graft after transplant nephrectomy
}

\author{
S.A. Prozorov \\ N.V. Sklifosovsky Research Institute for Emergency Medicine, \\ 3 Bolshaya Sukharevskaya Sq., Moscow 129090 Russia \\ Corresponding author: Sergey A. Prozorov, Dr. Med. Sci., Leading Researcher of the Department of Radiology, \\ N.V. Sklifosovsky Research Institute for Emergency Medicine, surgeonserge @ mail.ru
}

\section{Abstract}

Introduction. Vascular complications after transplant nephrectomy are rather rare. The aim of this article is to analyze the effectiveness of the stent-graft implantation in the treatment of pseudoaneurysm after transplant nephrectomy.

Clinical case. In the previous 23 years, a 50-year-old patient underwent 3 kidney transplantations from a cadaveric donor and 2 transplant nephrectomies. At 12 years after the left transplant nephrectomy he complained of abdominal pain and was admitted for a pulsating mass in his left pelvic region. Computed tomography angiography, ultrasonography, and arteriography were performed and showed a large pseudoaneurysm arising from the left external iliac artery. The stentgraft implantation solved the problem providing successful clinical and radiological results.

Conclusion. Treatment options in this case were open or endovascular techniques. Surgical revision associated with high risk of complications. Endovascular treatment had the benefits of a minimally invasive approach with low blood loss.

Keywords: renal transplantation, pseudoaneurysm, stent-graft

CONFLICT OF INTERESTS Author declares no conflict of interest

Financing The study was performed without external funding

For citation: Prozorov SA. Endovascular treatment of the pseudoaneurysm using stent-graft after transplant nephrectomy. Transplantologiya. The Russian Journal of Transplantation. 2020;12(4):311-318. (In Russ.). https://doi.org/10.23873/2074-0506-202012-4-311-318

КТ - компьютерная томография

ЛА - ложная аневризма

Введение

Трансплантацию почек применяют для лечения пациентов с терминальной стадией почечных заболеваний. Возможны различные сосудистые осложнения после трансплантации. Открытые реконструктивные операции в этих случаях связаныс возможностью дальнейших осложнений и потери трансплантата. Эндоваскулярные методы являются альтернативой для лечения сосудистых осложнений после трансплантации почки.

Суммарное количество всех сосудистых осложнений в нашем институте на 2013 г. составило 18 случаев на 429 трансплантаций почки 421 пациенту, в том числе тромбозы микроциркуляторного русла в результате развития острого гуморального или смешанного отторжения, резистентного к проводимой терапии (9 случаев); в 4 случаях острое отторжение привело к разрыву трансплантата и экстренной трансплантатэктомии; тромбоз магистральной артерии наблюдали у 1 больного; выявлены 2 случая развития стеноза почечной артерии; у 2 пациентов наблюдали венозный тромбоз [1].
УзИ - ультразвуковое исследование

ЦДК - цветное допплеровское картирование

D. Dimitroulis et al. [2] провели 1367 трансплантаций почки и отметили 38 серьезных сосудистых осложнений, которые привели к потере почечного трансплантата и 19 стенозов почечных артерий с последующим успешным лечением в большинстве случаев.

S. Aktas et al. [3] выявили 47 различных сосудистых осложнений $(2,55 \%)$ после 43 трансплантаций $(2,33 \%)$ у 1843 больных: наиболее часто стеноз почечной артерии (14), кинкинг почечной артерии (7), или одноименной вены (7), тромбоз почечной артерии (5), разрыв почечной вены (4), разрыв почечной артерии (3), тромбоз почечной вены (2), нарушение работы почечной артерии (2), обструкция почечной и подвздошной вен из-за давления лимфоцеле (1), обструкция почечной артерии и вены из-за давления гематомы (1) и артериовенозный свищ после чрескожной биопсии трансплантата (1).

M. Salehipour et al. [4] сообщили о 133 осложнениях $(8,86 \%)$ после 1500 операций трансплантации, в первую очередь - кровотечение $(6,1 \%)$, стеноз почечной артерии трансплантата $(1,7 \%)$, тромбоз почечной артерии $(0,6 \%)$ и тромбоз почечной вены $(0,5 \%)$. 
По данным A. Srivastava et al. [5], сосудистые осложнения имели место у 25 пациентов $(1,29 \%)$ на 1945 трансплантаций почки, чаще всего - стеноз артерии у 11 пациентов $(0,58 \%)$, тромбоз артерии - у $9(0,46 \%)$, тромбоз вены - у $3(0,15 \%)$ и образование ложной аневризмы - у $2(0,1 \%)$.

P. Orlić et al. [6] из 843 трансплантаций почки выявили последующие сосудистые осложнения у 57 пациентов $(6,76 \%)$, из них образование ложной аневризмы (ЛА) у $3(0,35 \%)$.

$\mathrm{Y}-\mathrm{H}$. Lin et al. [7] провели поиск по базам данных MEDLINE (1950 г. - 4 ноября 2016 г.) и EMBASE (1970 г. - 4 ноября 2016 г.) всех сообщений об экстраренальной ЛА или разрыве артерии после трансплантации почки и нашли 45 публикаций о случаях или сериях наблюдений, которые соответствовали критериям включения. Согласно этому обзору, уровень летальности составил 13,8\%; в 56,3\% зарегистрированных случаев потеря трансплантированной почки произошла без смертельного исхода. Среди выбранных 87 пациентов у $73(83,9 \%)$ сосудистые осложнения возникли в месте анастомоза. У большинства пациентов $(79,3 \%)$ были диагностированы ЛА, а у 18 пациентов $(20,7 \%)$ из 87 - разрыв артерии. При этом только у 33 пациентов $(37,9 \%)$ не отмечено развития патогенной инфекции. В случаях ее возникновения наиболее часто встречался С. albicans (24 больных).

Как следует из приведенных данных, образование ЛА после трансплантации почки является редким осложнением.

ЛА могут возникать также и после вынужденного удаления трансплантированной почки [8-13], при этом возникает необходимость проведения реконструктивных сосудистых операций. Альтернативу может представлять имплантация стент-графрта. Приводим клиническое наблюдение.

Клиническое наблюдение

Больной Д., 50 лет, переведен в институт с жалобами на наличие пульсирующего образования в левой подвздошной области. Появление припухлости пациент стал отмечать примерно в течение 50 дней, за несколько часов до поступления возникло резкое повышение температуры тела до $38^{\circ} \mathrm{C}$ и произошло увеличение в объеме образования в левой подвздошной области.

Из анамнеза известно, что пациент в течение многих лет проходил лечение, в ходе которого ему был произведен ряд операций в другом лечебном учреждении: в 1997 г. - аллотрансплантация трупной почки слева, в 2008 г. - трансплантатэктомия и повторная аллотрансплантация трупной почки справа, в 2009 г. - острый криз отторжения, в 2013 г. возвратная хроническая почечная недостаточность, по поводу которой проводили перитонеальный диализ. В 2016 г. выполнена повторная аллотрасплантация трупной почки слева (сформирован анастомоз с общей подвздошной артерией).

Диагноз: хронический гломерулонефрит, терминальная стадия хронической почечной недостаточности, состояние после повторной (третьей) аллотранспплантации трупной почки от 31.05.2016 г. Дисфункция трансплантата. Отторжение трансплатата почки по смешанному типу от 17.09.2019 г. Хронический вирусный гепатит В и С.

При осмотре в левой подвздошной области имело место пульсирующее образование размерами 4 х5 cм без изменения кожного покрова над ним.

По данным компьютерно-томографической (КТ) ангиографии отмечалось нарушение целостности левой наружной подвздошной артерии с формированием отграниченной гематомы

При триплексном сканировании по передней боковой стенке левой подвздошной артерии лоцировалось анэхогенное образование округлой формы с четкими ровными контурами размерами 5,5х4,0 cм с тромботическими массами по передней стенке, там же по переднебоковой стенке визуализировалось соустье с артерией, при цветном допплеровском картировании (ЦДК) определялся турбулентный кровоток с эффректом спонтанного контрастирования. Слева в забрюшинном пространстве в подвздошной области лоцировался трансплантат почки размерами 11,5х5,8 см, толщина паренхимы - до 1,7 см, чашечно-лоханочная система расширена за счет лоханки до 2,3 см, чашечек - до 1,3 см. При ЦдК кровоток определялся до капсулы. Заключение: эхопризнаки ложной аневризмы подвздошной артерии слева, эхо-признаки каликопиелэктазии трансплантированной почки.

В связи с наличием у больного разрыва и ЛА 21.02.2020 г. выполнено ангиографическое исследование (рис. а), а затем стенирование левой наружной подвздошной артерии - имплантирован баллоно-расширяемый стент-графт LifeStream фрирмы Bard ("C.R. Bard, Inc.", Ирландия): давление до 12 атм, диаметр расправленного стент-графта 10,2 мм, длина - 54,5 мм. (рис. b, с). ЛА выключена из кровотока, кровоток в левой нижней конечности - магистральный.

При контрольном ультразвуковом исследовании (УЗИ) на 4-е сут: магистральный тип кровотока по подвздошным и бедренным артериям, визуализирован стент-графт, к артерии интимно прилегает объемное образование неоднородной структуры средней и повышенной эхогенности размерами $6,4 \times 6,3$ см с анэхогенной зоной размерами $2,7 \times 2,9$ см, кровотока в образовании в режиме ЦдК нет. 


\section{BISE BEPORTS}

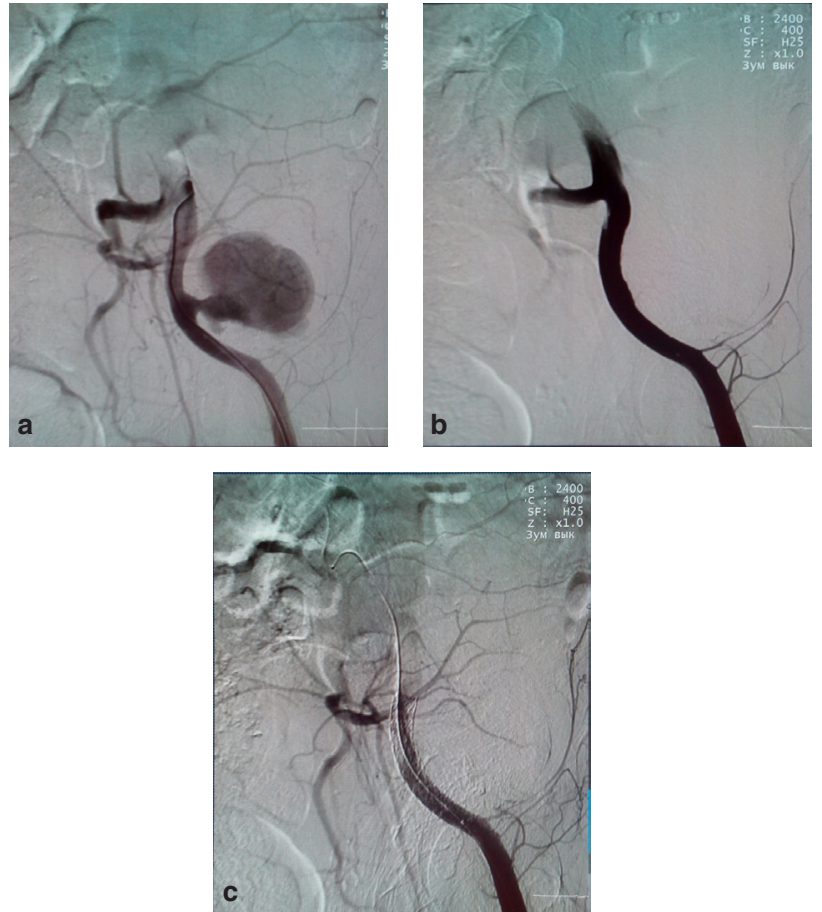

Рисунок. а-с. а - разрыв культи артерии трансплантированной почки с образованием ложной аневризмы, состояние после удаления трансплантата; b, c - выключение аневризмы из кровотока после имплантации стент-графта в наружную подвздошную артерию, фазы контрастирования

Figure. a-c. a, the artery stump rupture of the transplanted kidney with the pseudoaneurysm formation, the condition after the transplant nephrectomy; b, c, the aneurysm exclusion from the blood flow after the stent-graft implantation into the external iliac artery, contrast passage

Послеоперационное течение гладкое. Пациент выписан в удовлетворительном состоянии.

\section{Обсуждение}

У больного было произведено удаление нефункционирующего трансплантата почки (анастомоз артерии трансплантата с левой наружной подвздошной артерией). При этом был оставлен участок артерии трансплантата. В культе сосуда длительное время существовал турбулентный низкоскоростной кровоток. В соответствии с законами движения жидкостей Бернулли, чем ниже скорость движения жидкости, тем больше давление на стенку сосуда и наоборот, чем выше скорость движения жидкости, тем меньше давление на стенку сосуда. С течением времени (12 лет) происходила постепенная деградация стенки артерии. Возможными причинами также могут быть инфекция и отторжение культи сосуда [8-10].

Таким образом, в культе артерии после трансплантатэктомии существовало повышенное давление на стенку артерии с постепенной деградацией ее слоев, что и привело в конечном итоге к разрыву культи, образованию ЛА и необходимости эндоваскулярных диагностики и вмешательства.

Аневризмы после трансплантации почки могут возникать:

1. ЛА в месте сосудистого анастомоза $[8,9,11$, $14-28]$.

2. Истинные аневризмы артерии почечного трасплантата [29].

3. ЛА, диссекции в подвздошной артерии после операции [30-32].

4. ЛА подвздошной артерии или культи почечной артерии после трансплантатэктомии [8-13].

5. ЛА и артериовенозные соустья в паренхиме почки после биопсии [33-35].

ЛА обычно выявляют при УЗИ в режиме ЦдК, КТ-ангиография является подтверждающим тестом для планирования лечения [33]. Консервативное лечение возможно только в случаях небольших ЛА, без тенденции к увеличению размеров и без инфицирования [33, 34]. Оперативное или эндоваскулярное лечение необходимы для предотвращения/ликвидации разрыва и сохранения трансплантата.

Возможны различные варианты хирургического и эндоваскулярного лечения при ЛА. Выбор метода лечения зависит от анатомических особенностей артерии трансплантированной почки и ЛА, состояния больного и наличия инфекции. При этом эндоваскулярные методы имеют существенные преимущества, такие как меньшие травматичность вмешательства и количество осложнений. Однако все сообщения об эндоваскулярном лечении представляют собой либо единичные наблюдения, либо их небольшие серии.

Варианты малоинвазивного лечения:

1. Эмболизация ЛА [19, 23, 33-35].

Эмболизация различного типа микроспиралями возможна как при ЛА анастомозов $[19,23$, 33], так и суперселективная эмболизация при ЛА, возникших после биопсии $[34,35]$.

2. Эмболизация в сочетании с имплантацией стент-графта [11].

3. Эмболизация ЛА в сочетании с одномоментной дилатацией, стентирование выявленных стенозов артерии трансплантата [23, 28]. 
Так, G. Fananapazir et al. [23] выполнили больному эмболизацию ЛА спиралями, а затем имплантировали в ветви почечной артерии два коронарных стента с лекарственным покрытием.

Определенные технические сложности могут возникать при наличии ЛА с широкой шейкой. В таких случаях для решения проблемы используют два следующих метода.

4. Стент-ассистированная эмболизация $[17,18$, $21,29]$.

5. Использование при эмболизации страховочного баллонного катетера [26], который перекрывает шейку аневризмы и не дает сместиться спиралям в момент эндоваскулярной операции.

6. Имплантация стента, стент-графта [8-15, $20,23,27,30-33]$ как при ЛА анастомозов, так и при ЛА после нефрэктомии.

R. Vijayvergiya et al. [30] путем стентирования подвздошной артерии при ее ятрогенной диссекции восстановили кровоток по подвздошной артерии и артерии трансплантата. R.K. Peel et al. [32] имплантировали стент-графт при возникновении воспалительной неспецифической ЛА подвздошной артерии после трансплантации почки.

U.M. Bracale et al. [8, 9] проанализировали лечение 11 пациентов. У 5 (группа 1) возникли ЛА анастомозов почечных артерий трансплантата и подвздошных артерий и у 6 (группа 2) - ЛА подвздошных артерий после удаления трансплантированной почки. ЛА после нефрэктомии трансплантата являлись продолжением культи донорской почечной артерии. Эндоваскулярное лечение применили у 3 пациентов (1 - в группе 1, 2 - в группе 2); 8 больных перенесли открытое вмешательство. Стентирование проводили из ипсилатерального бедренного разреза под местной анестезией; использовали стент-графты диаметром 8-10 мм и длиной 50 или 60 мм. Спасение кровотока в конечности было достигнуто в $100 \%$ случаев. Ни у одного из пациентов не было отмечено развития поздней инфекции, неудачи при восстановлении сосудов или рецидива ЛА.

M.R. Smeds et al. [14] сообщили о случае эндоваскулярного лечения ЛА с помощью 2 «целующихся» (kissing) стент-графртов с полным исключением ЛА и с восстановлением отличного кровотока в трансплантированной почке и нижней конечности.

Y. Tshomba et al. [27] наблюдали больную с одновременной пересадкой почки и поджелудочной железы и возникшей ЛА анастомоза артерии почки с наружной подвздошной артерией диа- метром 9,5 см. Установленный стент-графт позволил восстановить нормальную перфузию органов.

B.C. McIntosh et al. [10] при ЛА после нефрэктомии трансплантата успешно имплантировали стент-графт.

7. Имплантация стент-графрта и введение в полость ЛА тромбина [16, 28].

J.A. Poels et al. [16] описали случай использования тромбина и стент-графрта для лечения ЛА при сохранении функции почки, a C. Kubal et al. [28] при ЛА диаметром 6 см ввели в полость тромбин, имплантировали стент-графрт и выполнили дилатацию внутриорганных ветвей баллонными катетерами диаметром 3 мм с быстрым улучшением фуннции почек.

8. Введение в полость аневризмы тромбина $[22,25,33]$.

Под ультразвуковым наведением производят чрескожную пункцию ЛА и вводят тромбин, в результате чего кровоток по ЛА прекращается.

В том случае, если не удается добиться эффекта в ходе одного вмешательства, возможно проведение повторной эндоваскулярной операции [17]: при лечении ЛА артерии почечного трансплантата первоначально был имплантирован стент от устья артерии трансплантата в среднюю треть наружной подвздошной артерии и выполнена эмболизация ЛА через микрокатетер (установлено 8 микроспиралей). При контрольном УЗИ было выявлено, что полный эффект эмболизации не достигнут. После установки вторым этапом дополнительных эмболизирующих спиралей при контрольном УЗИ кровотока в полости аневризмы не отмечено, сохранялся лишь небольшой участок с кровотоком в устье размерами $0,3 \times 0,3$ см.

Особую сложность представляет лечение больных с микотическими аневризмами $[11,12$, 15]. G. Zavos et al. [15] 3 пациентам с ЛА анастомозов установили стент-графты. Две из этих аневризм были вызваны грибковыми инфекциями. 8-недельная противогрибковая терапия оказалась успешной в предотвращении риска развития инфекции материала стент-графта: в сроки наблюдения от 2 мес до 3 лет признаков стеноза или иноекции не отмечалось.

P. Leonardou et al. [11] представили самую большую серию наблюдений из 5 больных с микотическими ЛА и комбинацию антибактериальной терапии, хирургического и интервенционных вмешательств. В 3 случаях эндоваскулярные операции провели при ЛА анастомозов и в 2 - после трансплантатэктомии. 4 пациентам имплантировали стент-графрты, одному выполнили эмболиза- 
цию и установили стент-графр, а после возникновения повторной ЛА имплантировали второй стент-графт; позже потребовались, кроме того, трансплантатэктомия и реконструкция подвздошных артерий. Еще в одном случае после нефрэктомии трансплантата имплантация трех стент-графтов не позволила решить проблему из-за их последующего иноицирования, в связи с чем произведены операция по удалению микотической ЛА и стентов и шунтирование.

D.D. Zhao et al. [12] сообщили о 2 случаях, когда после трансплантации почки у больных при профрилактике грибковой инфекции фрлуконазолом развилось кровотечение. У 1 пациента была выполнена нефрэктомия, но кровотечение повторилось, и в месте анастомоза с подвздошной артерией установили стент-графт, после чего кровотечение не повторялось. Во втором случае вследствие массивного кровотечения после трансплантации потребовались нефрэктомия трансплантата и исключение ЛА из кровотока с помощью стент-графта.

\section{Заключение}

Оставление после неорэктомии трансплантата длинной культи артерии донорской почки может приводить к образованию ложной аневризмы и ее разрыву. Использование стент-графта позволяет выключить ложную аневризму из кровотока, сохранить при этом магистральный кровоток, адекватное кровоснабжение нижней конечности и избежать открытой реконструктивной операции. Как видно, возможны различные варианты успешного применения эндоваскулярных методов лечения при образовании ложных аневризм после трансплантации почки или недрэктомии трансплантата.

\section{Литература / References}

1. Хубутия М.Ш., Пинчук А.В., Шмарина Н.В., Дмитриев И.В., Сторожев Р.В., Коков Л.С. и др. Сосудистые осложнения после трансплантации почки. Вестник трансплантологии и искусственных органов. 2013;15(4):31-39. Khubutiya MS, Pinchuk AV, Shmarina NV, Dmitriev IV, Storozhev RV, Kokov LS, et al. Vascular complications after kidney transplantation. Russian Journal of Transplantology and Artificial Organs. 2013;15(4):31-39. (In Russ.). https://doi.org/10.15825/1995-11912013-4-31-39

2. Dimitroulis D, Bokos J, Zavos G, Nikiteas N, Karidis NP, Katsaronis P, et al. Vascular complications in renal transplantation: a single-center experience in 1367 renal transplantations and review of the literature. Transplant Proc. 2009;41(5):1609-1614. PMID: 19545690 https://doi.org/10.1016/j.transproceed.2009.02.077

3. Aktas S, Boyvat F, Sevmis S, Moray G, Karakayali H, Haberal M. Analysis of vascular complications after renal transplantation. Transplant Proc. 2011;43(2):557-561. PMID: 21440760 https://doi.org/10.1016/j.transproceed.2011.01.007

4. Salehipour M, Salahi H, Jalaeian H, Bahador A, Nikeghbalian S, Barzideh E, et al. Vascular complications following 1500 consecutive living and cadaveric donor renal transplantations: a single center study. Saudi J Kidney Dis Transpl. 2009;20(4):570-572. PMID: 19587495

5. Srivastava A, Kumar J, Sharma S, Abhishek, Ansari MS, Kapoor R. Vascular complication in live related renal transplant: an experience of 1945 cases. Indian J Urol. 2013;29(1):42-47. PMID: 23671364 https://doi.org/10.4103/09701591.109983

6. Orlić P, Vukas D, Curuvija D, Markić D, Merlak-Prodan Z, Maleta I, et al. Pseudoaneurysm after renal transplantation. Acta Med Croatica. 2008;62(Suppl 1):8689. (In Croatian). PMID: 18578338

7. Lin $\mathrm{Y}-\mathrm{H}$, Liao $\mathrm{C}-\mathrm{H}$, Jiang B-J, Chen T-H. Early renal arterial rupture and arterial pseudoaneurysm in graft kidneys from the same deceased donor. Ci Ji Yi Xue Za Zhi. 2018;30(4):250254. PMID: 30305791 https://doi.org/ doi:10.4103/tcmj.tcmj_180_17

8. Bracale UM, Carbone F, del Guercio L, Viola D, D'Armiento FP, Maurea $\mathrm{S}$, et al. External iliac artery pseudoaneurysm complicating renal transplantation. Interact Cardiovasc Thorac Surg. 2009;8(6):654-660. PMID: 19289398 https://doi.org/10.1510/ icvts.2008.200386
9. Bracale UM, Santangelo M, Carbone F, Del Guercio L, Maurea S, Porcellini M, et al. Anastomotic pseudoaneurysm complicating renal transplantation: treatment options. Eur J Vasc Endovasc Surg. 2010;39(5):565-568. PMID: 20122855 https://doi.org/10.1016/j.ejvs.2009.12.010 10. McIntosh BC, Bakhos CT, Sweeney TF, DeNatale RW, Ferneini AM. Endovascular repair of transplant nephrectomy external iliac artery pseudoaneurysm. Conn Med. 2005;69(8):465466. PMID: 16270782

11. Leonardou P, Gioldasi S, Zavos G, Pappas P. Mycotic pseudoaneurysms complicating renal transplantation: A case series and review of literature. $J$ Med Case Rep. 2012;6:59. PMID: 22333365 https://doi.org/10.1186/1752-1947-6-59 12. Zhao DD, Huang $\mathrm{ZY}$, Hong LQ, Liao $\mathrm{T}$, Tang $\mathrm{YE}, \mathrm{Na} \mathrm{N}$, et al. Massive hemorrhage caused by fungal infections after donation-after-cardiacdeath kidney transplantation: Clinical features, prevention and treatment experience. Zhonghua Yi Xue Za Zhi. 2016;96(20):1570-1572. PMID: 27266684 https: / / doi.org / 10.3760 / cma.j.is sn.0376-2491.2016.20.005

13. Moosavi CA, Gujrathi SK, Friedman A, Fox D, Silberzweig JE. Endovascular Repair of Symptomatic Renal 
Transplant Site Pseudoaneurysm. Vasc Endovascular Surg. 2008-2009;42(6):607609. PMID: 18621888 https://doi. org/10.1177/1538574408320022

14. Smeds MR, Ofstein R, Peterson GJ, Peterson BG, Jacobs DL. Endovascular repair of a para-anastomotic pseudoaneurysm after renal autotransplantation: an alternative to open reconstruction. Ann Vasc Surg. 2013;27(1):110. e5-8. PMID: 23079504 https://doi. org/10.1016/j.avsg.2012.06.003

15. Zavos G, Pappas P, Kakisis JD, Leonardou P, Manoli E, Bokos J, et al. Endovascular repair as first-choice treatment of iliac pseudoaneurysms following renal transplantation. Transplant Proc. 2005;37(10):4300-4302. PMID: 16387102 https://doi.org/10.1016/j.transproceed.2005.11.034

16. Poels JA, Riley PL. Extrarenal transplant artery pseudoaneurysm: a combined therapeutic approach. Cardiovasc Intervent Radiol. 2008;31(2):404406. PMID: 17965910 https://doi. org/10.1007/s00270-007-9207-2

17. Верстова А.И., Коков Л.С., Пархоменко М.В., Пинчук А.В. Клинический случай эмболизации ложной аневризмы артерии почечного трансплантата. Российский Электронный Журнал Лучевой Диагностики. 2015;5(2)Прил.:231232. URL: http://www.rejr.ru/archiv. $\mathrm{html}$ [Дата обращения 10 июля 2020]. Verstova AI, Kokov LS, Parkhomenko MV, Pinchuk AV. Clinical case of embolization of a false aneurysm of the renal graft artery. Russian Electronic Journal of Radiology (REJR). 2015;5(2) Suppl.:231-232. Available at: http:// www.rejr.ru/archiv.html [Accessed July 10, 2020] (In Russ.)

18. Семитко С.П., Ванюков А.Е., Аналеев А.И., Азаров А.В., Климов В.П., Купкенова М.И. и др. Ложная аневризма артерии трансплантата почки: обзор литературы и пример успешного эндоваскулярного лечения. Диагностическая и интервениионная радиология. 2018;12(1):65-72. Semitko SP, Vanyukov AE, Analeev AI, Azarov AV, Klimov VP, Kupkenova MI, et al. False aneurysm of the kidney transplant artery: a review of the literature and an example of successful endascular treatment. Diagnostic and interventional radiology. 2018;12(1):65-72. (In Russ.). 19. Marie Y, Kumar A, Hinchliffe S, Curran S, Brown P, Turner D, et al. Treatment of transplant renal artery pseudoaneurysm using expandable hydrogel coils: A case report and review of literature. World J Transplant. 2018;8(6):232236. PMID: 30370233 https://doi. org/10.5500/wjt.v8.i6.232

20. Che H, Men C, Yang M, Zhang J, Chen P, Yong J. Endovascular repair of a transplant renal artery anastomotic pseudoaneurysm using the snorkel technique. J Vasc Surg. 2014;60(4):10521055. PMID: 23993437 https://doi. org/10.1016/j.jvs.2013.07.016

21. Favelier S, Kretz B, Tanter Y, Loffroy R. Stent-assisted detachable coil embolization of a late-onset widenecked anastomotic renal allograft artery pseudoaneurysm. J Vasc Surg. 2012;56(4):1131. PMID: 23026424 https:// doi.org/10.1016/j.jvs.2011.09.097

22. Reus M, Morales D, Vázquez V, Llorente $\mathrm{S}$, Alonso J. Ultrasound-guided percutaneous thrombin injection for treatment of extrarenal pseudoaneurysm after renal transplantation. Transplantation. 2002;74(6):882-884. PIID: 12364872 https://doi.org/10.1097/00007890200209270-00025

23. Fananapazir G, Hannsun G, Wright LA, Corwin MT, Troppmann C. Diagnosis and management of transplanted kidney extrarenal pseudoaneurysms: a series of four cases and a review of the literature. Cardiovasc Intervent Radiol. 2016;39(11):1649-1653. PMID: 27439622 https://doi.org/10.1007/ s00270-016-1425-z

24. Patil VV, Roytman M, Ames S, Beckerman W, Lookstein RA. Endovascular repair of renal artery anastomotic pseudoaneurysm following living donor kidney transplant. Cardiovasc Intervent Radiol. 2015;38(6):1640-1644. PMID: 26037091 https://doi.org/10.1007/ s00270-015-1136-x

25. Siu YP, Tong MKH, Leung KT, Kwan TH, Au TC, Cheung YK, et al. Renal artery pseudoaneurysm following renal transplantation and treatment by percutaneous thrombin injection. Hong Kong Med J. 2006;12(1):80-81. PMID: 16495597 26. Akgul E, Binokay F, Aikimbaev K, Aksungur EH. Extrarenal pseudoaneurysm of the arterial anastomosis in a renal transplant: Endovascular coil embolization with balloon remodeling technique. Ren Fail. 2011;33(4):452-455. PMID: 21529275 https://doi.org/10.3109 /0886022X.2011.568131

27. Tshomba Y, Apruzzi L, Kahlberg A, Baccellieri D, Vitale R, Chiesa R. Endovascular repair of delayed giant iliac pseudoaneurysm following simultaneous pancreas-kidney transplantation with preserved allografts function. $J$ Cardiovasc Surg (Torino). 2019;60(4):514517. PMID: 26505147 https://doi. org/10.23736/S0021-9509.16.08790-5

28. Kubal C, Cacciola R, Riley P, Ready A. Internal iliac artery pseudoaneurysm following renal transplant biopsy successfully treated with endovascular stenting and thrombosis: a case report. Transplant Proc. 2007;39(5):1676-1678. https://doi. org/10.1016/j.transproceed.2007.03.018 29. Li Z, Li Y, Jing Z, Feng R. Endovascular treatment for a true aneurysm of the transplant renal artery using noncovered stent-assisted coil embolization. Ann Vasc Surg. 2018;51:325.e5-325.e8. PMID: 29758329 https://doi.org/10.1016/j. avsg.2018.02.023

30. Vijayvergiya R, Sharma A, Singh S, Kanabar K. Endovascular treatment of an external iliac artery dissection causing early renal graft dysfunction. Ann Vasc Surg. 2019;59:312.e7-312.e9. PMID: 31009723 https://doi.org/10.1016/j. avsg.2018.12.108

31. Pérez P, Esteban C, Muchart J, Callejas JM. Endovascular resolution of iliac artery pseudoaneurysm in a transplanted patient. Nefrologia. 2004;24(6):596-599. (In Spanish). PMID: 15683034

32. Peel RK, Patel J, Woodrow G. Iliac artery false aneurysm following renal allograft: presentation with non-specific inflammatory response and treatment by endovascular stent graft. Nephrol Dial Transplant. 2003;18(9):1939-1940. PMID: 12937251 https://doi.org/10.1093/ndt/ gfg286

33. Glebova NO, Brooke BS, Desai NM, Lum YW. Endovascular interventions for managing vascular complication of renal transplantation. Semin Vasc Surg. 2013;26(4):205-212. PMID: 25220328 https://doi.org/10.1053/j.semvascsurg.2014.06.013

34. Zaragozano GR, García DAI, Cobos HMV, Yagüe RD. Postbiopsy giant pseudoaneurysm in renal transplant: treatment with embolization. Actas Urol Esp. 1998;22(3):267-271. (In Spanish). PMID: 9616940

35. Rivera M, Villacorta J, JiménezAlvaro S, Quereda C. Asymptomatic large extracapsular renal pseudoaneurysm following kidney transplant biopsy. Am $J$ Kidney Dis. 2011;57(1):175-8. PMID: 21184923 https://doi.org/10.1053/j. ajkd.2010.07.020 


\section{Ин формация обавторе}

Сергей Анатольевич Прозоров

Sergey A. Prozorov д-р мед. наук, ведущий научный сотрудник отделения лучевой диагностики ГБУЗ «НИИ скорой помощи им. Н.В. Склифосовского ДЗМ», http://orcid.org/0000-00029680-9722

\section{Information about the author}

Dr. Sci. (Med.), Leading Researcher of the Radiology Department, N.V. Sklifosovsky Research Institute for Emergency Medicine, http://orcid.org/0000-0002-9680-9722
Статья поступила в редакцию 23.07.2020;

одобрена после рецензирования 31.08.2020;

принята к публикации 30.09.2020
The article was received on July 23, 2020;

approved after reviewing August 31, 2020; accepted for publication September 30, 2020 